\title{
L'équipement des PME en systèmes ERP : une adoption guidée par les priorités stratégiques?
}

\author{
FRANCOIS DELTOUR \\ École des Mines de Nantes, \\ LEMNA
}

\author{
MEHDI FARAJALLAH \\ Ecole Nationale de Commerce \\ et de Gestion d'Agadir
}

\author{
VIRGINIE LETHIAIS \\ Telecom Bretagne, \\ Université de Bretagne Occidentale
}

et Université de Rennes 1

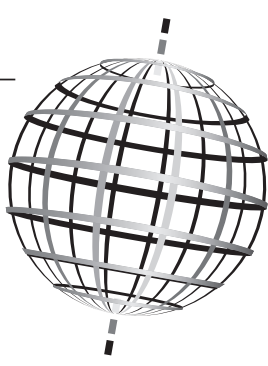

\section{RÉSUMÉ}

Pour les PME, investir dans un progiciel de gestion intégré - ERP - correspond à un investissement informatique majeur, assimilable à une décision stratégique. L'article teste l'influence des priorités stratégiques des PME sur leur choix d'utiliser ou non un système ERP. Une investigation auprès de 1977 PME montre que la recherche de réactivité est le seul positionnement stratégique augmentant la probabilité d'adopter un ERP. Les stratégies d'affaires centrées sur les caractéristiques des produits n'ont, elles, pas d'influence. De plus, l'investigation confirme que la taille, l'appartenance à un groupe ainsi que la maturité informatique des PME favorisent leur adoption des ERP.

Mots clés : ERP, priorités stratégiques, PME, adoption, économétrie.

\begin{abstract}
ERP is a major IT investment for SMEs, which can be considered as a strategic decision. The article tests how strategic priorities of SMEs influence their adoption of an ERP system. An investigation on 1977 firms shows that reactivity seeking is the only strategic positioning increasing the probability to adopt an ERP. Business strategies focused on the characteristics of the products have no influence. In addition, the investigation confirms that the size, the group membership and the IT maturity of SMEs lever ERP adoption.
\end{abstract}

Keywords: ERP, strategic priorities SME, adoption, econometrics.

\begin{abstract}
Resumen
Invertir en un software integrado ERP supone para las PYMES una inversión informática de importancia, asimilable a una decisión estratégica. El artículo analiza la influencia de las prioridades estratégicas de las PYMES en su decisión de utilizar o no un ERP. Una investigación realizada en 1977 PYMES muestra que la búsqueda de reactividad es el único posicionamiento estratégico que aumenta la probabilidad de adoptar un ERP. Las estrategias comerciales centradas en las características de los productos no influyen en la decisión. Tambien, el tamaño, el pertenecer a un grupo y la madurez informática favorecen la adopción de los ERP.
\end{abstract}

Palabras claves: ERP, prioridades estratégicas, adopción, econometria.
I informatisation croissante des entreprises se traduit (ordinateurs, accès internet) et par l'investissement dans les technologies les plus récentes : visioconférence, équipements mobiles, etc. (DGCIS, 2010). Au delà des équipements, l'investissement dans les logiciels de gestion est également primordial puisque ces logiciels agissent sur l'activité quotidienne des collaborateurs de l'entreprise. D'un point de vue historique, trois types de logiciels ont été adoptés, par vagues successives : les logiciels maison spécifiquement développés pour une fonction donnée de l'entreprise, puis les logiciels de marchés acquis et implantés autour d'un processus dédié, et enfin les progiciels de gestion intégrés, connus sous l'acronyme anglo-saxon ERP (Entreprise Resource Planning). Les ERP sont des logiciels du marché visant à permettre un fonctionnement totalement intégré des différents processus de l'entreprise (Segrestin et al., 2004). Aujourd'hui, les ERP équipent et structurent le fonctionnement de nombreuses grandes sociétés. D'après les statistiques européennes, les ERP ont été adoptés par $68 \%$ des grandes firmes (plus de 250 personnes). Pourtant, ils ne sont présents que dans $44 \%$

1. Données pour les 27 pays de l'Union Européenne, année 2012, toutes firmes hors secteur financier. Données disponibles sur http://appsso.eurostat.ec.europa.eu/ des sociétés de taille moyenne (50 à 249 employés) et dans $18 \%$ des petites entreprises (10 à 49 employés $)^{1}$. C'est auprès de ces catégories d'organisations que les enjeux économiques et organisationnels sont les plus forts, car les PME sont devenues le marché à conquérir pour les éditeurs de logiciels ERP alors que les bénéfices de ce type de logiciel intégré pour des organisations de taille réduite sont encore à démontrer.

D'un point de vue académique, le nombre de travaux consacrés à l'analyse de l'implantation des ERP est aujourd'hui conséquent (Schlichter et Kraemmergaard, 2010). Néanmoins, les petites et moyennes entreprises sont longtemps restées hors du champ de ces recherches. Ainsi, la revue de littérature de Aloini et al. (2007) sur les facteurs de risque du projet ERP regroupe un ensemble de 75 publications académiques parues depuis 1999 parmi lesquelles une seule publication est dédiée au contexte des PME. Plus récemment, Haddara et Zach (2012) ont proposé un état des lieux de la recherche émergente sur la question des ERP dans les PME. Leur analyse de la littérature amène au constat que l'adoption des ERP est étudiée sous l'angle de facteurs individuels, 
techniques, environnementaux ou bien organisationnels, mais que la dimension stratégique n'est jamais directement posée. Pourtant différents éléments plaident pour reconnaître le caractère stratégique des ERP pour les PME : de par les montants importants investis dans la mise en œuvre des ERP, ceux-ci représentent un engagement de long terme pour les entreprises, c'est-à-dire une décision de nature stratégique (Mourrain, 2007). De plus, le caractère fortement structurant des ERP vient bousculer l'ensemble du système de gestion très spécifique et souvent peu formalisé des petites structures (Segrestin et al., 2004; Haddara et Zach, 2012). Adopter un ERP implique donc de nombreux enjeux pour les PME, aussi bien financiers qu'organisationnels, qui en font un choix de dimension stratégique dans la vie de ces organisations. Le lien entre l'adoption de l'ERP et la conduite stratégique de la firme peut donc être posé. Ce lien a été étudié par Beard et Sumner (2004) et Ragowsky et Gefen (2008) qui montrent que les ERP ne peuvent être automatiquement considérés comme une source d'avantage concurrentiel stratégique. Notre recherche vise à compléter les travaux existants qui analysent l'adoption des ERP dans les petites structures en y intégrant des critères de nature stratégique. Cet objectif nécessite de prendre en compte les spécificités des stratégies menées par les PME, du fait de la faible formalisation de leur processus stratégique et de la forte influence de l'environnement (Marchesnay, 1991; Leitner et Güldenberg, 2010). Notre analyse est alors menée en mobilisant la notion de «priorités stratégiques » que nous relions à la décision d'adoption d'un ERP. Notre question de recherche est formulée de la façon suivante : existe-t-il une influence des priorités stratégiques des PME sur leur décision d'adopter un ERP?

Afin de répondre à notre question de recherche, l'article est structuré de la façon suivante : la première partie est consacrée à la construction du cadre d'analyse pour lequel nous identifions des priorités stratégiques à même d'inciter les PME à adopter un ERP. Nous relevons également les différents facteurs d'adoption précédemment identifiés dans la littérature sur l'adoption des ERP. La deuxième partie présente la collecte des données réalisée via une enquête portant sur l'utilisation des technologies de l'information (TI) dans 1997 PME. La méthode d'analyse statistique retenue est également exposée. Enfin, la dernière partie de l'article est consacrée à l'analyse et la discussion des résultats obtenus.

\section{Les déterminants de l'adoption des ERP}

Les systèmes ERP possèdent des caractéristiques très spécifiques qui ont des incidences fortes sur les conditions de leur adoption par les entreprises. L'ERP ou Progiciel de Gestion Intégré peut être défini comme une application «paramétrable, modulaire et intégrée, qui vise à intégrer et à optimiser les processus de gestion de l'entreprise en proposant un référentiel unique et en s'appuyant sur des règles de gestion standards» (Reix et al., 2011 p.97). Pour être considéré comme intégré, un progiciel de gestion doit remplir plusieurs conditions (Lemaire, 2003) : provenir d'un concepteur unique; garantir à l'utilisateur l'unicité de l'information, au moyen d'une base de données desservant l'ensemble des modules (un ERP implique au minimum la présence de deux modules fonctionnant ensemble avec les données partagées d'une base de données unique); répercuter toute saisie ou modification d'information dans l'ensemble des modules en temps réel; assurer la «traçabilité » des opérations de gestion pour en permettre l'audit; couvrir soit une fonction complète de gestion (gestion des ressources humaines, production...), soit la totalité du système d'information. La logique d'intégration des différents métiers de l'entreprise et la vision transversale qui en découle constituent donc deux effets majeurs attendus de l'ERP (El Amrani et al. 2006).

L'ERP représente aujourd'hui une option crédible pour les PME qui ambitionnent une rationalisation et une meilleure intégration de leur système d'information. Pourtant, l'implantation d'un ERP reste considérée comme un projet à enjeux forts (Deltour et Sargis Roussel, 2010; Deixonne 2011), par sa taille, son coût, ses fortes implications potentielles. Dans cette situation, certains contextes peuvent être identifiés comme plus favorables que d'autres pour qu'une PME adopte un ERP. De nombreux facteurs ont été identifiés dans la littérature comme pouvant affecter la décision d'adoption d'un ERP. Dans cet article, nous focalisons notre questionnement sur le rôle des choix stratégiques de la firme, qui constitue notre apport majeur à la littérature existante. Notre méthodologie intègre également des variables de contrôle, qui sont les facteurs d'adoption reconnus, que nous avons jugés les plus influents en ce qui concerne l'adoption des ERP.

\section{Adoption des ERP et PRIORITÉS STRATÉGIQUeS}

Le lien entre la politique en matière des TI et les choix stratégiques des entreprises est une préoccupation à la fois opérationnelle (Luftman et al., 2006) et académique (McLaren et al., 2011). Ainsi, Mclaren et al. ont récemment proposé de construire une mesure multi-niveaux de l'adéquation (' $f i t$ ') entre les stratégies compétitives des entreprises et leurs compétences organisationnelles issues de leurs systèmes d'information. Notre recherche, centrée sur les priorités stratégiques comme facteurs d'adoption, complète ce questionnement. Les priorités stratégiques suivies par une firme peuvent ainsi participer à comprendre sa politique en termes de technologies de l'information. Notre approche est alors contextualisée à une TI particulière - l'ERP - dans un contexte spécifique - les PME -, cette contextualisation apportant des enjeux particulièrement forts, aussi bien du point de vue organisationnel qu'économique.

\section{Les ERP et leur dimension stratégique pour les PME.}

La dimension stratégique des ERP peut se comprendre dans deux sens complémentaires. Tout d'abord, il y a l'idée qu'investir dans un ERP est une activité qui est de nature 
stratégique. Ensuite, il est à souligner que les fonctionnalités mêmes des ERP ne participent pas directement à la définition d'une stratégie, mais viennent plutôt soutenir les choix stratégiques qui ont été faits dans l'entreprise.

Investir dans un ERP est une activité stratégique des PME pour plusieurs raisons. Des montants importants sont investis dans la mise en œuvre des ERP, ce qui représente un engagement de long terme pour les entreprises. Dans les petites structures, la décision d'adopter un ERP revient d'ailleurs au dirigeant seul ou au comité de direction de l'entreprise (Mourrain, 2007). Les ERP sont alors implantés dans des petites organisations caractérisées par une faible formalisation des rôles, par une prédominance de l'ajustement mutuel et de la supervision directe, ou encore par des ressources limitées (Marchesnay, 1991). Comme le constatent Rougès et al. (2007, p.20) «la capacité d'une PME à absorber des échecs est limitée. Le choix d'investir dans les TI revêt donc, plus encore que dans les grandes entreprises, un enjeu stratégique majeur.»

Les fonctionnalités des ERP viennent soutenir les choix stratégiques des PME. En effet, les principales caractéristiques organisationnelles associées aux ERP sont celles de l'intégration fonctionnelle et de la standardisation. Les ERP sont un élément de renforcement de la transversalité dans l'entreprise, entre les différents métiers comme la vente, la production, la finance, les ressources humaines, les approvisionnements, etc. (El Amrani et al., 2006). De même, les processus prédéfinis dans les modules ERP sont plus ou moins fortement paramétrés lors de l'implantation. En basant le fonctionnement de leurs processus métiers sur l'ERP, les entreprises recherchent l'optimisation des différents flux nécessaires à leur activité (flux d'information, physiques, financiers) et cherchent à éviter différents écueils tels que les saisies multiples d'informations, les redondances et fréquentes incohérences d'informations ou le manque de données pour le pilotage des affaires. Ces bénéfices attendus en termes d'intégration fonctionnelle sont potentiellement importants pour l'entreprise, mais ne transforment pas nécessairement sa stratégie. Pour s'en convaincre, il est possible de se reporter au modèle de Venkatraman (1994) qui analyse la montée en puissance des systèmes d'information en proposant plusieurs étapes d'informatisation. Seules les étapes les plus élevées affectent la stratégie de l'entreprise (schéma 1).

Les caractéristiques de l'ERP le positionnent sur le modèle de Venkatraman aux niveaux intermédiaires 2 et 3, associés à l'intégration interne ainsi que la reconfiguration des processus de gestion (Schlichter et Kraemmergaard, 2010). Le niveau 4, lié aux relations avec les partenaires externes, est potentiellement concerné suite au récent déploiement de modules dédiés à la gestion de la chaîne logistique; on parle alors d'ERP II (Koh et al., 2011). Pourtant, la dimension des choix stratégiques comme le développement de nouvelles

\section{SCHÉMA 1}

\section{Place de l'ERP dans l'évolution des niveaux de définition et d'impact des systèmes d'information}

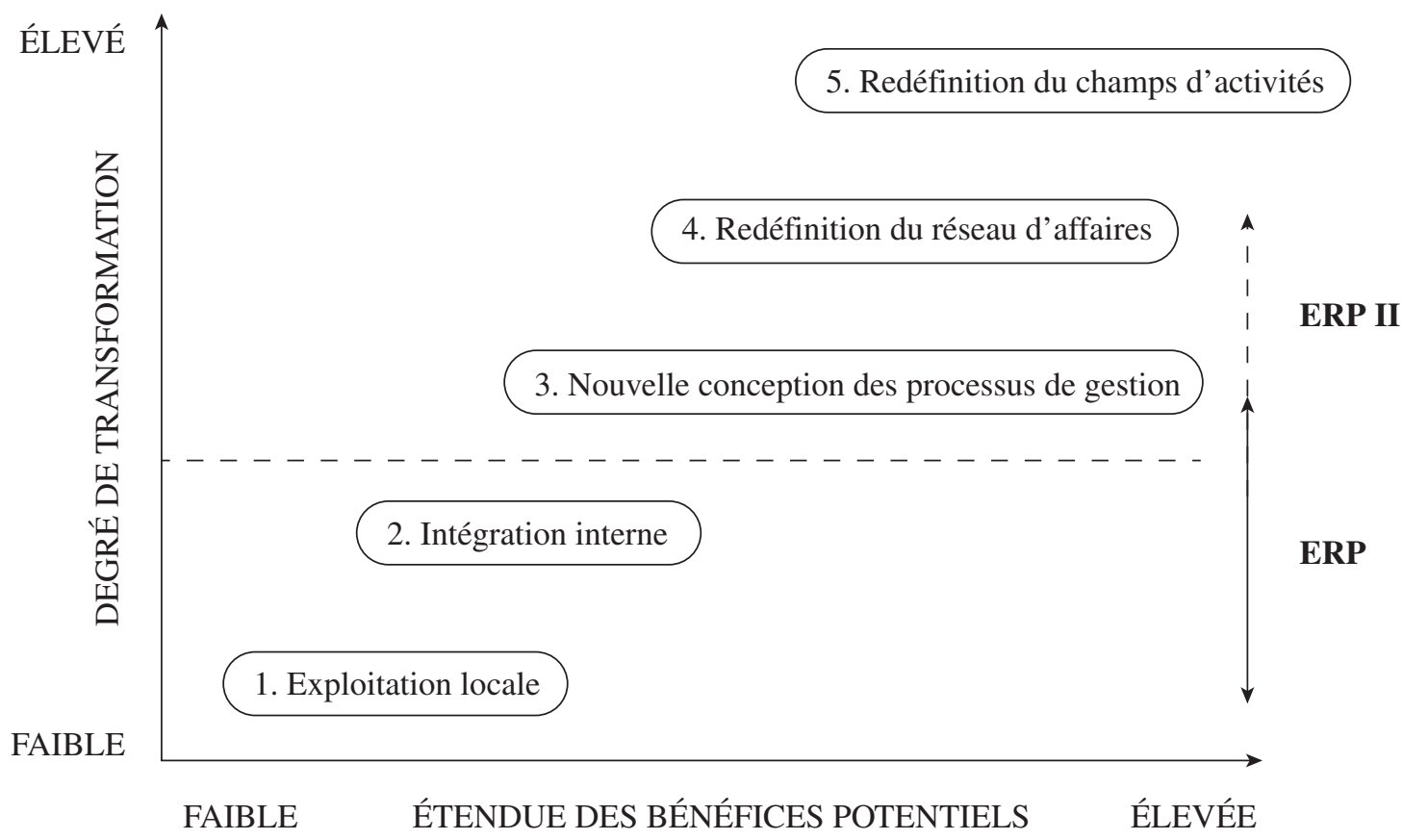

D'après le modèle de Venkatraman (adapté par Reix et al. 2011, p.266) 
activités se situe principalement au niveau 5. Le positionnement intermédiaire de l'ERP en fait un support potentiel indirect à d'autres TI dont les effets stratégiques sont eux, plus directs. Ainsi, Ash et Burn (2005) ont étudié comment la présence d'un ERP peut être un préalable à la mise en place d'une nouvelle stratégie web, de type e-business.

En définitive, nous soutenons l'idée que l'ERP n'est pas un déterminant de la stratégie des entreprises, mais vient soutenir la stratégie existante. Comme l'indique Mourrain (2011) au travers de douze études de cas d'ERP en PME, «Les entreprises de notre échantillon n'attendent pas $d u$ projet d'intégration des SI/TI un effet direct sur leur stratégie d'affaires » (p.10). Notre recherche se positionne bien dans une approche où l'ERP participe et soutient la mise en œuvre de choix stratégiques faits en amont. Suivant cette vision, l'ERP devient une ressource pour réaliser l'avantage concurrentiel visé (Kalling, 2003). Se pose alors la question de savoir si l'ERP est plus adapté à certaines stratégies qu'à d'autres.

\section{Approche de la stratégie des PME par les priorités stratégiques}

L'étude de la stratégie en tant qu'analyse des buts et des politiques générales mises en place dans les entreprises est un champ de recherche en soi. Dans la littérature en management stratégique, deux approches principales coexistent : la stratégie comme résultat et la stratégie comme processus. La première approche se centre sur l'étude des positions stratégiques au sein d'un secteur. La seconde se penche sur la manière dont la stratégie est construite, formulée et mise en œuvre. Notre recherche privilégie la première approche car il s'agit d'appréhender les choix de stratégie en lien avec la politique de système d'information. C'est donc une perspective porterienne qui est retenue. La stratégie y est envisagée comme un ensemble d'actions offensives ou défensives visant à créer un avantage concurrentiel permettant à l'entreprise de rendre profitables ses investissements (Porter, 1986). Pourtant, dans le contexte des PME, la question de l'établissement d'une stratégie prend un relief particulier qu'il faut prendre en compte : pour une PME, la question de l'existence d'une stratégie formalisée se pose (Marchesnay, 1991). Les douze études de cas menées par Mourrain (2011) rappellent que les PME ont des stratégies peu ou pas explicitées. De plus, comme le soulignent Rougès et al (2009b), il existe pour les PME un flou lié aux multiples dépendances à l'environnement de l'entreprise qui amène à une «double dynamique : la stratégie est imposée du haut [...] et elle émerge des décisions mises en place par les managers opérationnels $d u$ fait de leur environnement changeant» (p.65). Dans ce contexte, identifier clairement la stratégie suivie par une PME n'est pas aisé et certaines entreprises peuvent ne pas avoir de stratégie claire (Leitner et Güldenberg, 2010). Ceci nous amène à appréhender la stratégie au travers des différentes priorités stratégiques, plutôt que d'adopter une approche typologique classique. L'approche par les priorités stratégiques apparaît comme plus pertinente, car elle offre un moyen d'appréhender la manière dont est opérationnalisée la stratégie de l'entreprise.
La notion de priorités stratégiques a notamment été appliquée par Yen et Sheu (2004) dans le contexte des ERP. Les auteurs identifient dans la littérature les quatre critères suivants de priorité stratégique : prix, qualité (qualité de conception et qualité d'exécution), livraison (engagement à réaliser; vitesse de réalisation) et flexibilité (flexibilité de personnalisation; flexibilité de volume). Ces éléments sont utilisés dans des études de cas pour comprendre le processus d'implantation de l'ERP. De son côté, Stratman (2007) distingue deux formes d'orientations stratégiques : le focus stratégique sur les opérations (l'amélioration interne) et le focus stratégique sur le marché (l'amélioration vis-à-vis des clients et des concurrents). Nous reprenons ces deux catégories de priorités stratégiques en modifiant légèrement celle relative au focus sur le marché que nous élargissons à la notion de focus sur le produit/service offert au client, tel qu'appréhendé par Yen et Sheu (2004). Au final, nous considérons deux catégories de stratégies concurrentielles. La première, orientée produit, correspond à la recherche d'un avantage propre au bien ou service réalisé. Cet avantage peut notamment résider dans la nouveauté, la personnalisation du bien ou sa qualité technique. La seconde catégorie est centrée sur les conditions concurrentielles de l'offre. Elle peut se traduire par une plus grande réactivité ou un avantage en termes de prix.

\section{Les priorités stratégiques comme facteur d'adoption de l'ERP : les hypothèses}

La littérature sur les facteurs d'adoption des ERP s'est peu penchée sur la question du rôle de la stratégie ou des priorités concurrentielles. Certaines recherches excluent d'entrée cette variable, même si elles reconnaissent son rôle potentiel (Seddon et al., 2010). L'absence de réflexion stratégique est reconnue comme un risque majeur dans les phases initiales d'un projet ERP (Aloini et al., 2007). D'autres recherches se focalisent sur le rôle de la stratégie non pas sur la décision d'adoption des ERP, mais sur son processus d'implantation et sur les bénéfices qui peuvent en découler. C'est le cas des travaux de Maddox et Boyle (2007), Yen et Sheu (2004), Somers et Nelson (2003) et Kouki et al. (2009). Revenons sur les résultats obtenus par ces travaux afin d'alimenter nos hypothèses sur les priorités stratégiques comme facteurs d'adoption des ERP.

Les travaux de nature qualitative soulignent l'influence effective de la dimension stratégique dans l'implantation des ERP. Au travers de quatre études de cas (trois grandes entreprises et une PME), Maddox et Boyle (2007) identifient la non-prise en compte de la stratégie d'entreprise lors de l'implantation d'un ERP comme un élément altérant les bénéfices tangibles obtenus. De même, Yen et Sheu (2004) étudient cinq entreprises industrielles états-uniennes et taïwanaises et leur recherche confirme que l'implantation de l'ERP doit se faire en accord avec la stratégie concurrentielle de la firme. Dans leur conclusion, ils indiquent que : «Lorsque les firmes choisissent une stratégie de flexibilité ou de qualité d'exécution, ces priorités concurrentielles affectent les pratiques d'implantation en termes de centralisation, de paramétrage 
du logiciel, de partage d'information, de type et d'effort d'adaptation, et d'accès aux données. » (p. 218). Enfin, Kouki et al. (2009) montrent dans une étude de cas comparée de deux entreprises (une PME et une grande entreprise), que le processus d'assimilation des ERP passe, entre autre, au niveau stratégique par la reconnaissance au sein des entreprises de l'apport des ERP comme «un outil important pour l'efficience opérationnelle» (p.151). Cette contribution des ERP aux objectifs de performance opérationnelle des entreprises est également confirmée par les études quantitatives.

Plusieurs recherches quantitatives, comme celles de Raymond et Bergeron (2008) ou Sabherwal et Chan (2001) ont relié les comportements d'adoption de TI des entreprises à leur positionnement stratégique (sur la base des archétypes stratégiques de Miles et Snow). L'argumentation soutenue est que les entreprises de type «défenseur», qui se caractérisent par un objectif de production à bas coûts, une livraison rapide et par des économies d'échelles, sont des entreprises dont les «objectifs peuvent être atteints par l'usage de technologies comme l'EDI et l'ERP pour l'efficience de la chaîne de valeur interne et externe» (Raymond et Bergeron, 2008, p.582). A l'inverse, les entreprises dont la stratégie est de type «prospecteur» ou «analyste» investissent plutôt dans les TI aidant à la prise de décision, l'analyse des marchés ou des concurrents (Sabherwal et Chan, 2001). Ces résultats ont été obtenus auprès de firmes grandes ou moyennes (Sabherwal et Chan, 2001) mais aussi d'entreprises de petite taille (Raymond et Bergeron, 2008). Ils peuvent être repris et affinés dans le cadre de l'adoption spécifique des ERP en lien avec les priorités stratégiques des PME.

Nous avons précédemment distingué deux catégories de stratégies concurrentielles, l'une orientée autour des qualités du produit ou du service commercialisé (sa nouveauté, sa personnalisation, sa variété, ses qualités techniques), l'autre autour des conditions d'offre du produit (le positionnement prix et la recherche de réactivité client). Les éléments présentés nous permettent de formuler l'hypothèse que l'ERP ne soutient pas de façon directe la réalisation des priorités stratégiques centrées sur les produits et services. A l'inverse, l'ERP peut constituer un soutien fort pour les entreprises qui orientent leur stratégie vers l'efficience opérationnelle, en participant à l'optimisation de la production et donc à la baisse des coûts et à l'augmentation de la réactivité de ces PME. Nous pouvons alors formuler les deux hypothèses suivantes :

Hypothèse 1 : Les priorités stratégiques des PME centrées sur les conditions de l'offre influencent positivement l'adoption d'un ERP.

Hypothèse 2 : Les priorités stratégiques des PME centrées sur les produits n'ont pas d'influence sur l'adoption d'un ERP.

LES AUTRES DÉTERMINANTS DE L'ADOPTION DES ERP : PRISE EN COMPTE DE VARIABLES DE CONTRÔLE

Pendant la phase d'arbitrage qui précède l'adoption ou non d'un ERP, les entreprises analysent l'utilité d'un tel outil en fonction de plusieurs paramètres. Cette section fait état des déterminants de l'adoption d'un ERP que nous introduisons dans notre modèle de recherche comme variables de contrôle (schéma 2). Dans la littérature, ces facteurs ont été classés en plusieurs catégories. Même si les appellations de ces catégories diffèrent selon les auteurs, leurs contenus sont proches et évoquent à quelques exceptions près, des facteurs identiques explicatifs de l'adoption des ERP. Il s'agit classiquement de facteurs organisationnels, technologiques ou liés à l'environnement de l'entreprise (Tornatsky et Fleischer, 1993). Ces facteurs sont ici repris selon deux grandes catégories : les caractéristiques générales de la firme, d'une part, son profil informatique, d'autre part. Les facteurs environnementaux (par exemple liés à la pression concurrentielle) ne sont pas retenus ici car ils sont par nature intégrés dans la question du positionnement stratégique des entreprises. De même, une dimension complémentaire concernant la personnalité du dirigeant a aussi été mise en avant dans certains travaux sur l'adoption des ERP par les PME (Chang et al., 2010; Ramdani et al., 2009). De notre point de vue, le profil du dirigeant joue plus sur la politique globale de l'entreprise en termes d'investissement en technologie de l'information que sur la décision d'adopter spécifiquement un ERP. Cette influence potentielle est donc captée dans notre modèle par le degré d'informatisation global de l'entreprise.

\section{Les caractéristiques générales de la firme}

Taille et secteur d'activité. La complexité organisationnelle induite par l'augmentation de la taille d'une organisation peut la pousser à adopter un ERP. Cet effet taille est notamment démontré empiriquement par Mabert et al. (2003), Buonnano et al.(2005) ou Raymond et Uwizeyemungu (2007). Le choix d'adopter ou non un ERP peut également être affecté par le secteur d'activité, certains secteurs étant plus demandeurs de ressources, afin de gérer la complexité inhérente à leurs métiers (Raymond et al., 2006). C'est notamment le cas du secteur industriel où les processus de production peuvent être très morcelés, en comparaison par exemple au secteur de la vente où les activités se déroulent en beaucoup moins d'étapes (Buonnano et al., 2005). Conformément à ces différents travaux, nous testerons l'effet de la taille de l'entreprise et de l'appartenance au secteur industriel sur la probabilité d'adopter ou non un ERP.

Appartenance à un groupe. L'adoption d'un ERP est possible pour tout type d'entreprise, qu'elle appartienne à un groupe ou non. Balzi et al., (2006), dans une étude portant sur un échantillon de 687 PME suisses, trouvent que seulement $44 \%$ des 125 entreprises qui possèdent un ERP appartiennent à un groupe (holding ou autre). Pourtant, le fait d'appartenir à un groupe impose souvent aux unités qui le constituent de se coordonner. L'objectif est d'uniformiser les différentes étapes qui constituent les processus de production, que ce soit entre chaque unité et les dirigeants du groupe ou entre les différentes unités (Kocoglu et Moatty, 2010). Cet impératif de coordination engendre naturellement des flux 
additionnels d'information qui peuvent favoriser l'adoption des ERP. L'appartenance à un groupe peut aussi se traduire par une adoption «imposée» à la PME du système intégré adopté au sein du groupe (Dolmetsch et al., 1998). Parallèlement, Bunanno et al. (2005) ont montré que l'étendue géographique de la firme et notamment la présence à l'international, rendait les flux d'informations plus complexes et pouvait jouer positivement sur la décision d'adoption d'un ERP. Nous avons choisi d'introduire l'appartenance à un groupe comme facteur d'adoption des ERP, avec une nuance supplémentaire qui est l'étendue géographique du groupe, les flux entre les différentes unités du groupe étant potentiellement plus complexes dans le contexte international que dans un contexte national.

Qualification des salariés. Un autre facteur pouvant potentiellement influencer le recours à un ERP est le niveau de qualification des salariés. En effet, des travaux antérieurs font apparaître un lien positif entre le niveau de qualification des salariés de l'entreprise et l'adoption et l'utilisation des TI (Hollenstein, 2004, Galliano et Roux, 2007). Dans le cas particulier des ERP, Raymond et Uwizeyemungu (2007) montrent que la part des cadres dans les effectifs des entreprises joue positivement sur leur prédisposition à adopter un ERP.

\section{Le profil informatique de l'entreprise}

Niveau des usages informatiques. L'intégration d'un système ERP requiert un certain niveau de préparation pour l'entreprise, notamment via sa maturité technologique (Sammon et Adam, 2010). En particulier, l'existence dans la PME de différents systèmes informatisés proches ou antérieurs à l'ERP (logiciels d'aide à la production, logiciels de planification...) favorise l'adoption d'un ERP (Raymond et Uwizeyemungu, 2007). A l'inverse, l'absence d'expérience préalable d'un système d'information met une PME dans une situation de désinformation par rapport à ces nouvelles technologies et peut la décourager d'adopter un tel système.

Les compétences informatiques. Les compétences en informatique internes à l'entreprise peuvent affecter le choix d'adopter ou de ne pas adopter un système tel que l'ERP. Ces compétences peuvent prendre deux dimensions. La première est la familiarité de l'ensemble des salariés avec les outils informatiques qui peut influencer leur facilité à prendre en main un nouvel outil. Chang et al. (2010) montrent que les PME ayant des salariés familiarisés aux systèmes d'information sont significativement plus enclines à adopter des ERP. La seconde dimension est l'existence de compétences internes capables de veiller au bon fonctionnement du futur système. L'absence de compétences internes en TI dans les PME a été identifiée de longue date comme freinant l'adoption des TI (Cragg et King, 1993). A l'inverse, ces compétences présentes en entreprise favorisent l'adoption et le succès du projet ERP (Snider et al, 2009).

2. Bien que le questionnaire porte sur l'utilisation des TI, la part des directeurs informatiques dans les répondants reste très faible, la grande
En définitive, les variables mobilisées dans la recherche sont synthétisées dans le schéma 2.

\section{L'investigation quantitative auprès de PME françaises}

Les hypothèses de recherche formulées dans la partie conceptuelle sont testées empiriquement auprès d'entreprises ayant implanté un ERP. Plus précisément, nous construisons un modèle économétrique afin de déterminer les facteurs qui affectent la probabilité d'adopter ou non un ERP. Nous décrivons ici les données utilisées ainsi que le modèle économétrique et les variables que nous avons construites afin de tester empiriquement notre modèle de recherche.

\section{Collecte et TRAitement deS DONNÉES}

Les données utilisées sont issues d'une enquête menée en 2008 par le GIS Marsouin, dans le cadre de l'observatoire OPSIS (Observation et Prospective sur la Société de l'Information et les Services) (Marsouin, 2009). L'enquête porte sur l'utilisation des TI par les PME de la région Bretagne (France), de 10 à 250 salariés, appartenant aux secteurs de l'industrie, du commerce et des services (à l'exception des services publics). Le questionnaire, envoyé à toutes les entreprises concernées, pouvait être rempli sur document papier et renvoyé par courrier, ou bien rempli directement en ligne. Afin d'atteindre 2000 répondants, une administration complémentaire a été réalisée par téléphone. Les firmes ont été choisies afin d'obtenir une bonne représentativité finale des répondants en termes de localisation, de taille et de secteur d'activité, relativement au tissu économique régional. Le répondant est, dans la majorité des cas, le dirigeant de l'entreprise ou le responsable administratif ou/et financier ${ }^{2}$.

L'enquête repose sur un questionnaire général, qui interroge d'abord l'entreprise sur sa situation économique, et énumère ensuite une large palette d'équipements et leur utilisation possible à l'intérieur de la firme, ou bien avec des partenaires extérieurs (Marsouin, 2009). L'une des questions est «votre entreprise a-t-elle mis en place un ERP?». 254 entreprises (13\% des répondants) ont répondu positivement à cette question. Les réponses ont ensuite été filtrées grâce à deux types de questions additionnelles : une série de questions sur les modules ERP déployés dans les différentes fonctions de l'entreprise ainsi qu'une question sur le nom du logiciel et de l'éditeur de l'ERP. Un praticien «expert»a validé que le progiciel indiqué était bien de type ERP. Lorsque l'indication de l'éditeur ou du logiciel était incomplète ou absente, n'ont été retenus que les répondants qui déclaraient au moins deux fonctions de l'entreprise comme fonctionnant sous module ERP. Ces différents filtres ont amené à réduire le nombre d'entreprises utilisant effectivement un ERP à 84,

majorité des entreprises auxquelles s'adresse le questionnaire n'ayant pas de service informatique constitué. 


\section{SCHÉMA 2}

\section{Modèle général de la recherche}

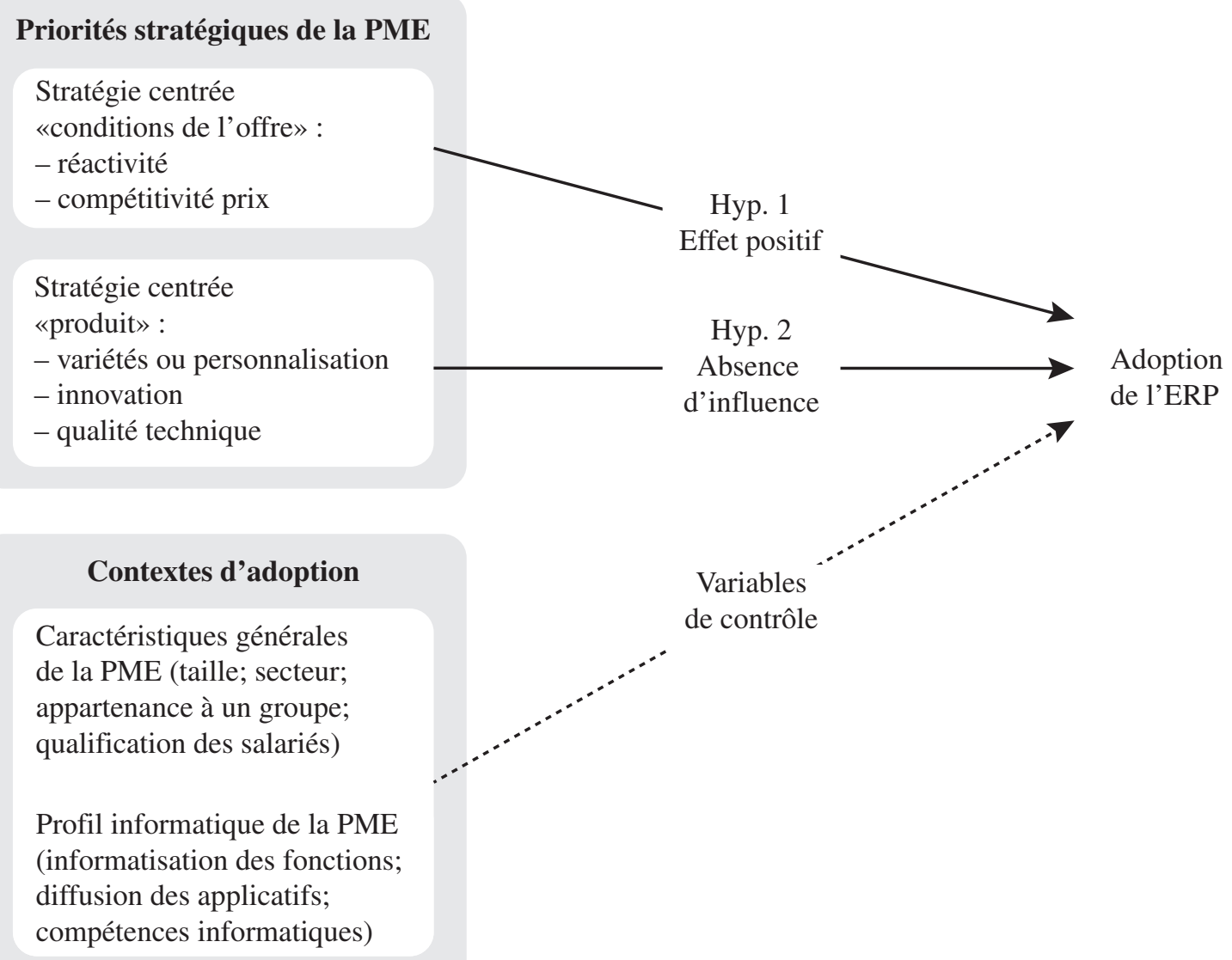

soit un taux d'adoption de 4,2\%. Cette réduction drastique est principalement due à la confusion faite par les répondants entre les ERP et d'autres applicatifs comme les logiciels de planification, de production ou bien financiers.

Après avoir exclu les questionnaires présentant trop de réponses manquantes, le nombre total d'entreprises dans notre échantillon s'élève à 1977, dont 81 ont adopté un ERP. Ce faible taux d'adoption en comparaison à d'autres études ${ }^{3}$ s'explique par notre volonté de nous focaliser sur les entreprises qui disposent effectivement d'un ERP.

\section{CHOIX DU MODÈLE ÉCONOMÉTRIQUe}

Afin de tester nos hypothèses sur les déterminants de l'adoption d'un ERP, nous utilisons un modèle de choix discret de type Logit (Cramer, 1991). Ce type de modèle permet de modéliser l'effet de plusieurs variables, indépendamment les

3. Le taux d'adoption obtenu est par exemple beaucoup plus faible que celui de la DGCIS (2010) pour qui, à date à peu près équivalente, les entreprises de 10 à 50 salariés sont $11 \%$ à posséder un ERP, et $36 \%$ unes des autres, sur la probabilité de choisir une alternative parmi plusieurs. Dans notre cas, l'objectif étant de mesurer l'influence des priorités stratégiques sur la décision d'adopter un ERP (plutôt que de ne pas en adopter), nous utilisons un modèle Logit binomial. Dans ce modèle, la variable «Adopt $\mathrm{ERP} »$ qui est notre variable dépendante vaut 1 si la PME choisit d'adopter un système ERP et 0 sinon et nous évaluons l'influence des priorités stratégiques des PME, ainsi que d'autres facteurs, sur la probabilité d'adopter un ERP.

\section{Mesure des variables}

Nous considérons trois grandes catégories de variables qui peuvent affecter le choix d'adoption des PME : les variables de positionnement stratégique, les caractéristiques générales de la firme ainsi que son profil informatique.

dans les moyennes entreprises. Mais ces chiffres ne font l'objet d'aucun filtrage ou vérification, tel que réalisé dans notre étude, et sont plus à rapprocher du chiffre de nos $13 \%$ «bruts» trouvés avant vérification. 


\section{Les priorités stratégiques de l'entreprise}

Les entreprises ont été interrogées sur leur choix concernant «les éléments clés du positionnement de l'entreprise» avec cinq modalités de réponses possibles : la personnalisation, la nouveauté, la qualité technique, la réactivité et enfin, la compétitivité prix (plusieurs réponses possibles). Les trois premiers facteurs traduisent une orientation stratégique de l'entreprise centrée sur le produit, alors que les deux derniers peuvent être associés à une stratégie centrée sur les conditions de l'offre. L'importance de chacun de ces facteurs dans la stratégie de l'entreprise est intégrée dans le modèle sous la forme d'une variable dichotomique qui prend la valeur 1 si l'entreprise a identifié ce facteur comme un élément clé de son positionnement stratégique et la valeur 0 dans le cas contraire.

\section{Les caractéristiques générales de la firme}

L'enquête nous informe sur les principales caractéristiques de l'entreprise interrogée. La taille de l'entreprise est intégrée dans le modèle sous la forme d'une variable continue : le nombre de salariés. La variable activité industrielle prend la valeur 1 lorsque les entreprises appartiennent au secteur industriel et 0 sinon. Conformément à notre analyse concernant l'appartenance à un groupe, nous avons construit une variable prenant trois modalités : 0 si l'entreprise n'appartient pas à un groupe, 1 si elle appartient à un groupe d'étendue régionale ou nationale, et 2 si elle appartient à un groupe international. Enfin, la variable qualification des salariés prend la valeur 1 lorsque la part des salariés de l'entreprise ayant le baccalauréat est supérieure à $10 \%$ et 0 sinon.

\section{Le profil informatique de l'entreprise}

Le profil informatique de la firme est caractérisé par trois variables distinctes : le niveau de préparation de l'entreprise à l'adoption, la familiarité des utilisateurs aux outils informatiques et enfin l'existence de compétences internes. Le questionnaire nous renseigne, pour l'ensemble des fonctions possibles au sein d'une entreprise (comptabilité et finance, gestion des ventes, des achats, des stocks, logistique/distribution, ressources humaines...) si chacune est assurée en interne, externalisée ou inexistante et, lorsqu'elle est réalisée en interne, si elle est informatisée, soit avec un logiciel du marché, soit avec un module d'un ERP, soit avec une «solution maison » (développée en interne). La variable taux d'informatisation des fonctions, qui représente le nombre de fonctions informatisées dans l'entreprise sur le nombre de fonctions assurées en interne, est une variable continue dont la valeur est comprise entre 0 et 1 .

D’autre part, les entreprises ont été interrogées sur leur utilisation des huit outils suivants : un système informatisé d'échange de données (EDI), un intranet, des listes de

4. Un rapport de cote égal à $\mathrm{x}$ implique que la probabilité d'adopter un ERP est multipliée par x pour la modalité considérée par rapport à la modalité de référence. diffusions interne, des agendas partagés, des espaces de travail partagés, des logiciels de définition ou de suivi des processus (workflow), des logiciels «métier» (spécifiques à l'activité de l'entreprise), des certificats électroniques. Notre objectif étant de mesurer un effet d'apprentissage «global» des utilisateurs, nous avons fait le choix d'introduire dans le modèle une variable agrégée, qui somme ces applicatifs utilisés. La variable diffusion des applicatifs, dont la valeur est comprise entre 0 et 8 , donne une mesure des savoirs faire et compétences acquises par les utilisateurs.

Enfin, l'existence de compétences en interne susceptibles de veiller au bon fonctionnement de l'activité est mesurée par la variable compétences informatiques, qui peut prendre trois modalités : 2 si l'entreprise dispose d'un service informatique constitué, 1 si elle dispose d'au moins un salarié à mi-temps ou plus dédié à l'informatique (mais pas de service informatique constitué) et 0 si elle ne dispose d'aucune de ces ressources en interne.

Les fréquences associées à chacune de ces variables, sur l'échantillon total, sur l'échantillon des entreprises ayant adopté un ERP et sur l'échantillon des firmes n'ayant pas adopté d'ERP, sont présentées en annexe.

\section{Résultats et analyse}

Le tableau 1 présente les coefficients des différentes variables, la valeur du t de Student (indiquée entre parenthèses) et les rapports de côte («odds ratios») associés à ces variables. Les étoiles indiquent le degré de significativité des variables. Pour les variables multinomiales, la modalité de référence est notée «Réf». Pour un modèle Logit, le coefficient estimé d'une variable explicative ne nous renseigne que sur le sens de la relation qui existe entre cette variable et la variable expliquée. Il est alors usuel et recommandé d'interpréter les rapports de côtes pour juger de l'influence de chaque variable explicative ${ }^{4}$.

\section{LA STRATÉGIE DE «RÉACTIVITÉ » COMME SEUL DÉTERMINANT STRATÉGIQUE DE L'ADOPTION DES ERP}

Nous avons posé deux hypothèses complémentaires selon lesquelles les priorités stratégiques des PME centrées sur les conditions de l'offre influencent positivement l'adoption d'un ERP alors que les priorités stratégiques centrées sur les produits n'ont pas d'influence.

Notre régression valide en partie notre hypothèse de l'influence des stratégies centrées sur l'offre. En partie, car seul un positionnement stratégique lié à la réactivité a un impact sur la probabilité d'adopter un ERP. En effet, si une PME décide d'adopter un tel positionnent, cela multiplie par 2 (odds ratio $=2.063$ ) les chances qu'elle adopte un système 
TABLEAU 1

Résultats du modèle Logit sur l'adoption ou non d'un ERP

\begin{tabular}{|c|c|c|c|c|}
\hline & \multirow{2}{*}{\multicolumn{2}{|c|}{ Variables explicatives }} & \multicolumn{2}{|c|}{ Adopter un ERP } \\
\hline & & & Coef.estimé & Odds ratios \\
\hline \multirow{5}{*}{$\begin{array}{l}\text { Priorités stratégiques } \\
\text { de la PME }\end{array}$} & \multicolumn{2}{|c|}{ Variétés et personnalisation } & $\begin{array}{c}-0.0338 \\
(-0.12)\end{array}$ & .966 \\
\hline & \multicolumn{2}{|l|}{ Nouveauté } & $\begin{array}{l}0.0274 \\
(0.09)\end{array}$ & 1.027 \\
\hline & \multicolumn{2}{|c|}{ Qualité Technique } & $\begin{array}{c}-0.0447 \\
(-0.17)\end{array}$ & .956 \\
\hline & \multicolumn{2}{|l|}{ Réactivité } & $\begin{array}{l}0.725 * * * \\
(2.83)\end{array}$ & $2.063 * * *$ \\
\hline & \multicolumn{2}{|c|}{ Compétitivité prix } & $\begin{array}{l}0.302 \\
(1.21)\end{array}$ & 1.352 \\
\hline \multirow{6}{*}{$\begin{array}{l}\text { Caractéristiques } \\
\text { générales de la PME }\end{array}$} & \multicolumn{2}{|l|}{ Taille } & $\begin{array}{c}0.00437 * \\
(1.87)\end{array}$ & $1.004 *$ \\
\hline & \multicolumn{2}{|c|}{ Qualification des salariés } & $\begin{array}{l}0.763 * \\
(1.94)\end{array}$ & $0.466^{*}$ \\
\hline & \multicolumn{2}{|c|}{ Activité industrielle } & $\begin{array}{l}0.546^{*} \\
(1.79)\end{array}$ & $1.725^{*}$ \\
\hline & \multirow{3}{*}{$\begin{array}{l}\text { Appartenance } \\
\text { à un groupe }\end{array}$} & $\begin{array}{l}\text { Appartenance à un groupe } \\
\text { international }\end{array}$ & $\begin{array}{c}1.144 * * * \\
(3.59)\end{array}$ & $3.139 * * *$ \\
\hline & & $\begin{array}{l}\text { Appartenance à un groupe national ou } \\
\text { régional }\end{array}$ & $\begin{array}{c}0.562^{*} \\
(1.89)\end{array}$ & $1.754^{*}$ \\
\hline & & PME indépendante & Réf & Réf \\
\hline \multirow{5}{*}{$\begin{array}{l}\text { Profil informatique } \\
\text { de la PME }\end{array}$} & \multirow{2}{*}{\multicolumn{2}{|c|}{ Taux d'informatisation des fonctions }} & $\begin{array}{l}2.501 * * * \\
(3.86)\end{array}$ & $12.190 * * *$ \\
\hline & & & $\begin{array}{l}0.165 * * * \\
(2.80)\end{array}$ & $1.179 * * *$ \\
\hline & \multirow{3}{*}{$\begin{array}{l}\text { Compétences } \\
\text { informatiques }\end{array}$} & Service informatique & $\begin{array}{c}0.748 * * \\
(2.36)\end{array}$ & $2.113 * *$ \\
\hline & & Personnel informatique & $\begin{array}{c}0.674 * * \\
(2.23)\end{array}$ & $1.963 * *$ \\
\hline & & Pas compétence informatique & Réf & Réf \\
\hline Constante & & & $\begin{array}{l}-7.091 * * * \\
(-10.42)\end{array}$ & \\
\hline Nb d'observations & \multicolumn{4}{|c|}{1977} \\
\hline Log de vraisemblance & \multicolumn{4}{|c|}{-266.847} \\
\hline Significativité & \multicolumn{4}{|c|}{$(* 0.10 * * 0.05 * * * 0.01),()$ t de Student } \\
\hline
\end{tabular}


ERP. La recherche - voire l'impératif - de réactivité vis-à-vis des clients constitue donc un élément d'adoption des ERP. Ce résultat va dans le même sens que le constat de Kocoglu et Moatti (2010), qui indiquent que, grâce aux ERP, «les dirigeants sont alors équipés pour contrôler l'entreprise et la piloter. Le suivi de l'activité leur permet de réagir rapidement aux aléas liés à l'approvisionnement ou aux marchés et d'adapter les ressources au niveau d'activité.» (p.52). L'ERP répond à la recherche de réactivité voulue par les entreprises, du fait des caractéristiques de cet outil centrées sur la transversalité, l'intégration fonctionnelle ainsi que sur la standardisation du fonctionnement interne de la firme. Notons que, à la suite des études de cas menées par Yen et Sheu (2004), il n'existe pas de mode d'implantation typique des ERP pour des entreprises qui suivent un objectif de réactivité. Néanmoins ces derniers indiquent que lorsqu'une flexibilité en volume est recherchée (s'adapter à différents niveaux de production), l'ERP est implanté suivant un objectif de partage de l'information, avec une politique d'adaptation et de paramétrages avancés du système.

L'absence d'influence de la priorité stratégique de compétitivité-prix sur l'adoption d'ERP est inattendue au regard de nos hypothèses. Pourtant, l'idée que les PME opérant sur un marché sensible au prix ou plus concurrentiels sont plus prédisposées à adopter un ERP a été précédemment retenue et confirmée (Raymond et Uwizeyemungu, 2007; Chang et $a l ., 2010)$. Le raisonnement initial était qu'une stratégie de compétitivité-prix sur le marché était portée en interne par une politique d'efficience opérationnelle basée sur des processus de production favorisant les économies d'échelles et la réduction des coûts. L'adoption d'un ERP pouvait alors favoriser cette efficience opérationnelle et donc répondre à la priorité stratégique de compétitivité-prix. Cette absence d'influence peut aussi être expliquée par un découplage possible entre le positionnement commercial de l'entreprise et sa politique interne d'optimisation des coûts portée par l'ERP : rechercher un avantage concurrentiel en vendant un même bien ou service moins cher que ses concurrents n'est pas systématiquement relié à une optimisation des processus de production. D'autres méthodes peuvent ainsi permettre d'atteindre cette compétitivité-prix, comme par exemple la mise en place d'une organisation réduite par un recours à la sous-traitance (Gray et al., 2009) comportant un nombre restreint de services et de personnel, ou bien le recours à des approvisionnements en matières premières moins coûteux que les concurrents.

Enfin, en accord avec nos hypothèses, la régression confirme qu'aucun des positionnements stratégiques centrés sur le produit n'a d'impact sur la probabilité d'adoption de l'ERP : les déterminants de nouveauté, personnalisation, variété et qualité n'ont pas d'influence sur le choix d'adopter un ERP. Les questions liées à la manière dont la PME peut innover, se différencier ou améliorer ses produits sont indépendantes de la question de l'adoption d'un ERP.

D’une manière générale, l'influence relativement faible des priorités stratégiques sur le choix d'adopter un ERP peut être mise en perspective par rapport à la situation des PME. Leur taille modeste et leur orientation vers des préoccupations opérationnelles plutôt qu'une réflexion stratégique (Mourrain, 2007) éclairent nos résultats. D'ailleurs, Ragowski et Gefen (2008) constatent que le faible volume d'activité et le nombre réduit d'employés présents dans une entreprise sont deux facteurs expliquant que l'ERP est uniquement considéré comme un élément d'efficience opérationnelle (où l'ERP est vu comme un système «critique dans les opérations de l'entreprise») et non pas comme un élément d'avantage compétitif (où l'ERP est un système «au service de la compétition sur le marché»).

\section{LES CARACTÉRISTIQUES GÉNÉRALES ET INFORMATIQUES DE PME COMME DÉTERMINANTS DE L'ADOPTION DES ERP}

Tout d'abord, concernant les caractéristiques générales de la PME, nos travaux valident un certain nombre de résultats présents dans la littérature.

Conformément aux travaux antérieurs, la taille de l'entreprise joue positivement sur la probabilité d'adopter un ERP. La faiblesse du rapport de côte (qui est de 1,004) peut s'expliquer par notre choix d'avoir gardé cette variable sous sa forme quantitative : l'arrivée d'un seul salarié supplémentaire dans une PME influence faiblement les chances de celle-ci d'adopter un ERP.

Le type d'activité est également une des caractéristiques qui influence positivement l'adoption, puisqu'il apparaît qu'une PME qui a une activité industrielle a 1,7 fois plus de chance d'avoir un ERP qu'une PME qui a une activité de service. Ce résultat confirme les conclusions d'études antérieures, notamment celle de Buonnano et al. (2005). De plus, il met en avant la spécificité du système ERP par rapport à d'autres systèmes d'information : Ramdni et al. (2009) ont en effet montré, avec une méthodologie similaire, sur un échantillon de 102 entreprises petites et moyennes, que le secteur d'activité (notamment l'appartenance au secteur industriel) n'influençait pas l'adoption des systèmes intégrés (enterprise systems) au sens large (qui regroupent les ERP, le Customer Relationship Management, le Supply Chain Management et le e-procurement).

Concernant la variable d'appartenance à un groupe, il apparaît que chacune des modalités est significative. Limpact de cette variable combinée confirme les résultats obtenus par de travaux précédents qui ont traité l'appartenance à un groupe et l'étendue géographique de manière indépendante (Kocoglu et Moatty, 2010; Dolmetsch et al., 1998; Buonnano et al., 2005), mais permet de plus de mesurer les effets relatifs de chacun de ces facteurs. Il est en effet intéressant de noter que l'étendue du groupe à l'international a un impact plus fort que la simple appartenance à un groupe. En effet, le fait d'appartenir à un groupe régional ou national multiplie par 1,75 les chances d'adopter un ERP alors que le fait que le groupe soit international la multiplie par 2, puisque le rapport de côte pour la modalité «appartenance à un groupe 
international» est deux fois plus important que pour la modalité «appartenir à un groupe non international» $(3,14$ contre $1,75)$.

Enfin, le niveau de qualification des salariés, apparaît comme un facteur favorisant la probabilité d'adoption de cet outil sur notre échantillon de PME. En effet pour les PME dans lesquelles $10 \%$ ou plus des salariés ont un niveau d'étude supérieur au bac, les chances d'adopter un ERP sont deux fois plus importantes que pour les firmes dans lesquelles le niveau d'éducation est inférieur. Nous rejoignons ici un résultat mis en évidence par Raymond et Uwizeyemungu (2007) sur l'impact positif du pourcentage de cadres dans les effectifs de l'entreprise sur sa prédisposition à adopter un ERP.

Pour ce qui est du profil informatique de l'entreprise, on constate que toutes les variables explicatives introduites dans le modèle sont significatives et ont un coefficient positif. Conformément aux travaux antérieurs, la maturité technologique de la firme (Sammon et Adam, 2010; Raymond et Uwizeyemungu, 2007), la diffusion des usages (Chang et $a l ., 2010)$ et la présence de compétences internes en informatique (Cragg et King, 1993; Snider et al, 2009) jouent positivement sur la probabilité d'adopter un ERP. Les rapports de côte montrent toutefois des différences notables quant au poids de chaque variable, notamment au niveau de l'impact du taux d'informatisation. En effet, une première lecture des résultats montre que le rapport de côte pour cette variable est de 12,2. Ce chiffre est élevé car comme pour l'effectif des salariés, la variable de taux d'informatisation a été conservée sous sa forme continue. Le résultat peut être interprété de la manière suivante : quand une PME passe d'aucune fonction informatisée à toutes ses fonctions informatisées, cela multiplie par 12,2 les chances que celle-ci adopte un ERP. L'impact de l'indice TI qui combine les différents usages est également positif mais le poids de cette variable est moins élevé : notre modèle indique que l'augmentation d'un point de l'indice multiplie par 1,18 les chances qu'une PME adopte un ERP. Enfin, en ce qui concerne les compétences informatiques en interne, notre régression montre qu'une PME qui possède des compétences informatiques en interne a plus de chance d'adopter un ERP qu'une PME qui n'en possède pas. Il est de plus intéressant de noter que, si la présence de compétences informatiques multiplie par 2 environ les chances d'adopter un ERP, la différence entre la présence d'au moins un salarié à mi-temps dédié à l'informatique et l'existence d'un service informatique constitué est faible (1,963 contre 2,113).

\section{Conclusion}

L'ERP constitue un outil puissant d'intégration, de partage des informations et de fluidification des processus au sein des organisations (El Amrani et al. 2006; Kocoglu et Moatti, 2010). Si l'organisation totalement intégrée par son système d'information demeure un «mythe » (pour reprendre l'expression de Segrestin et al. 2004), des bénéfices sont néanmoins retirés de l'adoption et l'usage des ERP. Ces bénéfices, qui ont pu profiter en premier lieu aux grandes entreprises, sont aujourd'hui à portée de main des petites et moyennes entreprises. Pourtant la contrepartie est de taille pour les PME : la complexité, l'investissement et le coût représentés par l'adoption d'un ERP font de ce système un fait majeur de la vie de ces sociétés, affectant l'ensemble de l'organisation, à commencer par le quotidien de très nombreux salariés. Afin de comprendre les facteurs pouvant expliquer le choix pour une PME de recourir à un ERP, nous avons mobilisé la littérature existante tout en nous interrogeant sur le rôle d'un facteur spécifique : la stratégie mises en œuvre par la PME.

A notre question de recherche qui pose la question de l'influence des priorités stratégiques des PME sur leur décision d'adopter un ERP, notre échantillon d'entreprises de 10 à 250 salariés apporte finalement une réponse modérée : d'un côté, les entreprises axant leurs priorités stratégiques autour des caractéristiques des produits ou services vendus (leur nouveauté, leur personnalisation, leur qualité) ne sont pas spécifiquement enclines à adopter un ERP, celui-ci ne répondant pas directement aux priorités stratégiques fixées. D’un autre côté, les entreprises stratégiquement orientées vers une forme d'excellence opérationnelle (bas coûts, forte réactivité) ne sont enclines à adopter un ERP que lorsque l'objectif de «réactivité» est affiché. Les questions stratégiques jouent donc un rôle dans l'adoption des ERP, mais au même titre que d'autres variables qui sont ici significativement influentes, comme l'appartenance à un groupe international, la taille de l'entreprise, le degré d'informatisation et l'existence de compétences informatiques internes.

Notre recherche apporte un éclairage nouveau, complémentaire aux recherches antérieures, sur les conditions d'adoption des ERP, dans le contexte spécifique des PME. Plusieurs implications managériales peuvent être mises en avant, dans une logique de contingence de l'adoption des systèmes d'information. Pour les PME souhaitant adopter un ERP, notre recherche peut aider au diagnostic stratégique à mener préalablement à l'adoption, notamment sur l'importance que l'entreprise accorde à sa réactivité comme facteur d'avantage concurrentiel. Pour les prestataires spécialisés dans l'implantation d'ERP, nos résultats rappellent l'importance des différences de profils de leurs prospects : les déterminants mis en avant permettent d'identifier les prospects qui sont les plus à même d'adopter un système ERP.

Enfin, certaines limites à ce travail peuvent être relevées. D'un point de vue empirique, notre validation a été menée sur un faible nombre d'entreprises adoptantes, ce qui a limité les analyses économétriques. D'un point de vue méthodologique, notre recherche est confrontée à des questions de temporalités, qui sont importantes aussi bien dans les recherches en stratégie qu'en systèmes d'information : sur la base du questionnaire, nous avons évalué le lien entre le positionnement stratégique actuel est le choix de l'ERP. Or, la mise en place d'un ERP prend du temps et elle est soumise à des jeux politiques qui peuvent aussi remettre en cause la 
stratégie de l'entreprise. Cette dimension n'est pas ici prise en compte. Elle requiert des méthodologies autres, plutôt de nature qualitative. C'est ce qu'ont notamment investigué Lee et Myers (2004) sous forme ethnographique où le cas étudié a montré que «la firme possédait un ERP qui ne reflétait plus les objectifs stratégiques de l'entreprise» (p.356). Enfin, d'un point de vue conceptuel, nous pouvons souligner deux limites actuelles, qui constituent des pistes pour des investigations futures.

Une première piste porte sur la prise en compte des spécificités des logiciels ERP. Adopter un ERP, c'est adopter un certain nombre de modules fonctionnant de façon intégrée. La question du nombre et du type de modules utilisés par les entreprises n'est pas analysée dans cette recherche. Un approfondissement peut donc porter sur l'analyse de différents profils d'ERP adoptés par les PME. Cette prise en compte des différents modules est amorcée par Hitt et al. (2002), qui distinguent différents profils d'ERP afin d'analyser les bénéfices qui en sont retirés par 1117 grandes entreprises américaines.

Une seconde piste de recherche viserait à compléter la démarche de contingence entre la stratégie et l'ERP en questionnant la performance qui peut en découler. On se rapprocherait alors de la perspective de l'alignement stratégique de Venkatraman (1994). Cet alignement stratégique facteur de performance a été testé positivement par Kefi (2011) auprès d'un échantillon principalement composé de PME. Ce lien avec la performance serait d'autant plus intéressant à étudier que la littérature associant TI et performance dans le contexte des PME a débouché sur des résultats ambigus (Rougès $e t$ al., 2007). L'analyse des effets des ERP se trouverait alors confrontée à de nouvelles limites, liées plus généralement à la question de la dilution des impacts organisationnels des TI (Reix et al. 2011, pp. 417-419) : dilution des effets dans le temps, dilution par le passage d'une approche technique à une approche organisationnelle puis in fine financière. Autant de défis d'analyse qui concernent aussi bien les petites que les grandes entreprises.

\section{Références}

Aloini, D.; Dulmin, R.; Mininno, V. (2007). «Risk management in ERP project introduction: Review of the literature», Information \& Management, vol. 44, $\mathrm{n}^{\circ}$ 6, p. 547-567.

Ash, C.G.; Burn, J.M. (2003). «A strategic framework for the management of ERP enabled e-business change», European Journal of Operational Research, vol.146, n², p. 374-387.

Balzi, E.; Equey, C. (2006). Etude du comportement des PME/ PMI suisses en matière d'adoption de système de gestion intégré : entre méconnaissance et satisfaction, Genève : CRAG Haute école de gestion de Genève, 76 p.

Beard, J.W.; Sumner, M. (2004). «Seeking strategic advantage in the post-net era: Viewing ERP systems from the resourcebased perspective», The Journal of Strategic Information Systems, vol. 13, $\mathrm{n}^{\circ} 2$, p. 129-150
Buonanno, G.; Faverio, P.; Pigni, F.; Ravarini, A.; Sciuto, D.; Tagliavini, M. (2005). «Factors affecting ERP system adoption. A comparative analysis between SMEs and large companies», Journal of Enterprise Information Management, vol. $18, n^{\circ} 4$, p. $384-426$.

Chang, S.I.; Hung, S.Y.; Yen, D.; Lee, P.J (2010). «Critical factors of ERP adoption for small-and medium-sized enterprises: An empirical study », Journal of Global Information Management, vol. 18, $\mathrm{n}^{\circ}$ 3, p. 82-106.

CragG, P.B.; King, M. (1993). «Small-firm computing: Motivators and inhibitors », MIS Quarterly, vol. 17, n 1, p. 47-60.

Cramer, J. S. (1991). The logit model for economists. London and New York, Edward Arnold.

DGCIS (Direction générale de la compétitivité et de l'industrie et des services) (2010), Tableau de bord des TIC et du commerce électronique, Ministère de l'Economie, 32 p.

DeIXonne, J.L. (2011). Piloter un projet ERP, Editions Dunod, Paris. 3ème edition, $304 \mathrm{p}$.

Deltour, F.; Sargis Roussel, C. (2010). «L'intégration des connaissances par les équipes projets ERP : deux études de cas en $\mathrm{PME} »$, Systèmes d'Information et Management, vol. $15, \mathrm{n}^{\circ} 1$, p. 9-34.

Dolmetsch, R.; Huber, T.; Fleisch, E.; Oesterle, H. (1998). «R/3 implementation in small and midsize companies », dans Dolmetsch R., Huber T., Fleisch E., Oesterle H. (Eds.), Accelerated SAP: 4 Case Studies, Institute for Information Management, Universität St. Gallen, p. 51.

El Amrani, R.; Rowe, F.; Bidan, M.; Geffroy-Maronnat, B.; MarciniaK, R. (2006). "Les effets de la stratégie de déploiement des PGI sur la vision transversale de l'entreprise», Revue Française de Gestion, vol. 32, n 168- 169, p. 267-285.

Galliano, D.; Roux, P. (2008). «Organisational motives and spatial effects in Internet adoption and intensity of use: Evidence from French industrial firms », Annals of Regional Science, vol. 42, $\mathrm{n}^{\circ} 2$, p. 425-448.

Gray, J.V.; Roth, A.V.; Tomlin, B. (2009). «The influence of cost and quality priorities on the propensity to outsource production», Decision Sciences, vol. 40, n 4, p. 697-726.

Haddara, M.; Zach, O. (2012). "ERP systems in SMEs: An extended literature review», International Journal of Information Science, vol. $2, \mathrm{n}^{\circ}$ 6, p. 106-116.

Hiтt, L.M.; Wu, D.J.; Zhou, X. (2002). «Investment in enterprise resource planning: Business impact and productivity measures », Journal of Management Information Systems, vol. 19, $\mathrm{n}^{\circ} 1, \mathrm{p} .71-98$.

Hollenstein, H. (2004). "The determinants of the adoption of ICT », Structural Change and Economics Dynamics, vol. 15, $\mathrm{n}^{\circ} 3$, p. 315-342.

Kefi, H. (2011). «Processus organisationnels et systèmes d'information et de communication : alignement et performance», La Revue des Sciences de Gestion, vol. 5, $\mathrm{n}^{\circ} 251$, p. $189-200$.

Kocoglu, Y.; Moatty, F. (2010). «Diffusion et combinaison des TIC au sein des entreprises en 2006 : les réseaux, la gestion des données et l'intégration par les ERP», Réseaux, vol. 28, $\mathrm{n}^{\circ} 162$, p. 37-71. 
Koh, S.C.L.; GunaseKaran, A.; Goodman, T. (2011). «Drivers, barriers and critical success factors for ERP II implementation in supply chains: A critical analysis », Journal of Strategic Information Systems, vol. 20, n 4, p. 385-402.

KALLING, T. (2003). «ERP systems and the strategic management processes that lead to competitive advantage», Information Resources Management Journal, vol. 16, n 4, p. 46-68.

Kouki, R.; Pellerin, R.; Poulin, D. (2009). «Antecedents of ERP assimilation: The cases of a medium-sized and a large manufacturing company», in Information and Communication Technology and Small and Medium Sized Enterprises: From Theory to Practice, Poulin, D., Tran, S. (eds), Cambridge Scholars Press.

LEE, J.C.; MYERs, M.D. (2004). «Dominant actors, political agendas, and strategic shifts over time: A critical ethnography of an enterprise systems implementation », The Journal of Strategic Information Systems, vol. 13, $\mathrm{n}^{\circ}$ 4, p. 355-374.

Leitner, K.-H.; GüLdenberg, S. (2010). «Generic strategies and firm performance in SMEs: A longitudinal study of Austrian SMEs», Small Business Economics, vol. 35, n 2, p. 169-189.

Lemaire, L. (2003). Systèmes de gestion intégrés : des technologies à risques?, Editions Liaisons, $142 \mathrm{p}$.

Luftman, J.; Kempaiah, R.; Nash, E. (2006). «Key issues for IT executives $2005 »$, MIS Quarterly Executive, vol. $5 \mathrm{n}^{\circ} 2$, p. 80-99.

Mabert, V.A.; Soni, A.; Venkataramanan, M.A. (2003). «The impact of organization size on enterprise resource planning (ERP) implementations in the US manufacturing sector», Omega, vol. 31, n 3, p. 235-246.

Maddox, D.; Boyle, G. (2007). «Information technology performance and process: Four case studies », Perspectives on Irish Productivity, chapter 22, p. 350-363.

Marchesnay, M. (1991). «La PME : une gestion spécifique», Économie rurale, $\mathrm{n}^{\circ}$ 206, p. 11-17.

Marsouin (2009) Pénétration, diffusion et usages des TIC dans les PME, Document en ligne disponible à l'adresse : http:// www.marsouin.org/spip.php ?article292

McLaren, T.; Head, M.; Yuan, Y.; Chan, Y. (2011). «A multilevel model for measuring fit between a firm's competitive strategies and information systems capabilities », MIS Quarterly, vol. $35, n^{\circ} 4$, p. $909-929$.

Mourrain, A. (2007). L'investissement stratégique en pré-implémentation dans le cadre d'un projet d'intégration des systèmes d'information : le cas des PME, Thèse de doctorat, Université de Bretagne Occidentale, $452 \mathrm{p}$.

Mourrain, A. (2011). «La décision de lancement d'un projet d'intégration des SI/TI et l'alignement stratégique. Etude de cas de PME», Actes du 16ème Colloque de l'AIM, St Denis de la Réunion.

PorTER, M. (1986). L'avantage concurrentiel, Paris, InterEditions, $647 \mathrm{p}$.

Ragowsky, A.; Gefen, D. (2008). «What makes the competitive contribution of ERP strategic », Database for Advances in Information Systems, vol. 39, ${ }^{\circ}$ 2, p. 33-49.

Ramdani, B.; Kawalek, P.; Lorenzo, O. (2009). «Predicting SMEs' adoption of enterprise systems », Journal of Enterprise Information Management, vol. 22, $\mathrm{n}^{\circ}$ 1, p. 10-24.
RAYMOND, L.; BERGERON, F. (2008). «Enabling the business strategy of SMEs through e-business capabilities: A strategic alignment perspective», Industrial Management \& Data Systems, vol. $108, \mathrm{n}^{\circ} 5$, p. $577-595$.

Raymond, L.; Rivard, S., Jutras, D. (2006). «Evaluating readiness for ERP adoption in manufacturing SMEs », International Journal of Enterprise Information Systems, vol. 2, $\mathrm{n}^{\circ} 4$, p. 1-17.

RAYMOND, L.; UwIZEYEMUNGU, S. (2007). «A profile of ERP adoption in manufacturing SMEs», Journal of Enterprise Information Management, vol. 20, $\mathrm{n}^{\circ}$ 4, p. 487-502.

Reix, R.; Fallery, B.; Kalika, M.; Rowe, F. (2011). Systèmes d'information et management des organisations, 6ème édition, Vuibert, $496 \mathrm{p}$.

Rougès, J.F.; Poulin, D.; D’Amours, S.; Montreuil, B. (2007). «Les Technologies de l'Information et de la Communication, levier de performance pour les PME : revue d'une littérature ambiguë», Actes de la Conférence Internationale e-Commerce \& Gouvernance de l'Internet, Sousse, 19 \& 20 octobre.

Rougès, J.F.; Poulin, D.; D’Amours, S.; Montreuil, B. (2009a). «Relationship between SME performance and information and communication technology", in Information and Communication Technology and Small and Medium Sized Enterprises: From Theory to Practice (Poulain D. and Tran S. eds.), p. 25-47.

Rougès, J.F.; Poulin, D.; Bergeron, F.; Cimon, Y.B. (2009b). «The alignment of IT in SMEs and its contribution to performance: Research directions", in Information and Communication Technology and Small and Medium Sized Enterprises: From Theory to Practice (Poulain D. and Tran S. eds.), p. 48-68.

Sabherwal, R.; Chan, Y.E. (2001). «Alignment between business and IS strategies: A study of prospectors, analyzers, and defenders », Information Systems Research, vol. 12, n 1, p. 11-33.

Sammon, D.; Adam, F. (2010). «Project preparedness and the emergence of implementation problems in ERP projects », Information \& Management, vol. 47, $\mathrm{n}^{\circ} 1$, p. 1-8.

Schlichter, B.R.; Kraemmergaard, P. (2010). «A comprehensive literature review of the ERP research field over a decade», Journal of Enterprise Information Management, vol. $23 n^{\circ} 4$, p. $486-520$.

Seddon, P.B.; CALVert, C. (2010). «A multi-project model of key factors affecting organizational benefits from enterprise systems », MIS Quarterly, vol. 34, n² 2, p. 305-311.

Segrestin, D.; Darréon, J.L.; Trompette, P. (éds) (2004). «Le mythe de l'organisation intégrée : les progiciels de gestion», dossier thématique, Sciences de la Société, nº 61, février.

Snider, B.; Da Silveira, G.; Balakrishnan, J. (2009). «ERP implementation at SMEs: Analysis of five Canadian cases », International Journal of Operations \& Production Management, vol. 29, $\mathrm{n}^{\circ}$ 1, p. 4-29.

Somers, T.M.; Nelson, K.G. (2003). «The impact of strategy and integration mechanisms on enterprise systems value: Empirical evidence from manufacturing firms », European Journal of Operational Research, vol. 146, n 2, p. 352-364.

Stratman, J. K. (2007). «Realizing benefits from enterprise resource planning: Does strategic focus matter?», Production and Operations Management, vol. 16, n 2, p. 203-216. 
Tornatsky, L.G.; Fleischer, M. (1993). The process of technological innovation, Lexington Books, Lexington, MA.

Venkatraman, N. (1994). «IT enabled business transformation: From automation to business scope redefinition», Sloan Management Review, vol. 35, $\mathrm{n}^{\circ}$ 2, p. 73-87.

Yen, H.J.; SHeu, C. (2004). «Aligning ERP implementation with competitive priorities of manufacturing firms: An exploratory study», International Journal of Production Economics, vol. $92, \mathrm{n}^{\circ} 3$, p. $207-220$.

\section{ANNEXE}

\section{Statistiques descriptives de l'échantillon}

\begin{tabular}{|c|c|c|c|c|c|}
\hline & & & $\begin{array}{c}\text { PME sans } \\
\text { ERP } \\
(n=1896)\end{array}$ & $\begin{array}{c}\text { PME avec } \\
\text { ERP } \\
(\mathbf{n}=\mathbf{8 1})\end{array}$ & $\begin{array}{c}\text { Echantillon } \\
\text { complet } \\
(\mathbf{n}=1977)\end{array}$ \\
\hline \multirow{5}{*}{$\begin{array}{l}\text { Priorités } \\
\text { stratégiques } \\
\text { de la PME }\end{array}$} & \multicolumn{2}{|c|}{ Variétés et personnalisation (\% d'entreprises) } & 45,0 & 48,1 & 45,1 \\
\hline & \multicolumn{2}{|c|}{ Nouveauté (\% d'entreprises) } & 19,5 & 24,7 & 19,7 \\
\hline & \multicolumn{2}{|c|}{ Qualité technique (\% d'entreprises) } & 64,5 & 65,4 & 64,5 \\
\hline & \multicolumn{2}{|c|}{ Réactivité (\% d'entreprises) } & 37,3 & 60,5 & 38,2 \\
\hline & \multicolumn{2}{|c|}{ Compétitivité prix (\% d'entreprises) } & 40,0 & 50,6 & 40,4 \\
\hline \multirow{6}{*}{$\begin{array}{l}\text { Caractéristiques } \\
\text { générales de la PME }\end{array}$} & \multicolumn{2}{|c|}{ Taille de l'entreprise (nombre moyen de salariés) } & 30 & 63 & 32 \\
\hline & \multicolumn{2}{|c|}{$\begin{array}{l}\text { Qualification des salariés (\% d'entreprises ayant } \\
\text { au moins de } 10 \% \text { de salariés bacheliers) }\end{array}$} & 69,0 & 90,1 & 69,9 \\
\hline & \multicolumn{2}{|c|}{$\begin{array}{l}\text { Activité industrielle (\% d'entreprises dans } \\
\text { l'industrie) }\end{array}$} & 13,2 & 27,2 & 13,7 \\
\hline & \multirow{3}{*}{$\begin{array}{l}\text { Appartenance à un } \\
\text { groupe } \\
\text { (\% d'entreprises) }\end{array}$} & $\begin{array}{l}\text { Appartenance à un } \\
\text { groupe international }\end{array}$ & 8,8 & 30,9 & 9,7 \\
\hline & & $\begin{array}{l}\text { Appartenance à un } \\
\text { groupe national ou } \\
\text { régional }\end{array}$ & 17,1 & 30,9 & 17,7 \\
\hline & & PME indépendante & 74,0 & 38,3 & 72,6 \\
\hline \multirow{5}{*}{$\begin{array}{l}\text { Profil informatique } \\
\text { de la PME }\end{array}$} & \multicolumn{2}{|c|}{$\begin{array}{l}\text { Taux d'informatisation des fonctions (\% moyen } \\
\text { de fonctions informatisées) }\end{array}$} & 67,5 & 89,8 & 68,4 \\
\hline & \multicolumn{2}{|c|}{ Diffusion des applicatifs (nombre moyen) } & 2,6 & 4,6 & 2,7 \\
\hline & \multirow{3}{*}{$\begin{array}{l}\text { Compétences } \\
\text { informatiques } \\
\text { (\% d'entreprises) }\end{array}$} & Service informatique & 20,3 & 28,4 & 20,6 \\
\hline & & Personnel informatique & 13,9 & 37,0 & 14,9 \\
\hline & & $\begin{array}{l}\text { Pas de compétence } \\
\text { informatique }\end{array}$ & 65,8 & 34,6 & 64,5 \\
\hline
\end{tabular}


Copyright of Management International / International Management / Gestión Internacional is the property of Management International and its content may not be copied or emailed to multiple sites or posted to a listserv without the copyright holder's express written permission. However, users may print, download, or email articles for individual use. 